\title{
ON SELMER GROUPS OF ABELIAN VARIETIES OVER $\ell$-ADIC LIE EXTENSIONS OF GLOBAL FUNCTION FIELDS
}

\author{
A. BANDINI AND M. VALENTINO
}

\begin{abstract}
Let $F$ be a global function field of characteristic $p>0$ and $A / F$ an abelian variety. Let $K / F$ be an $\ell$-adic Lie extension $(\ell \neq p)$ unramified outside a finite set of primes $S$ and such that $\operatorname{Gal}(K / F)$ has no elements of order $\ell$. We shall prove that, under certain conditions, $\operatorname{Sel}_{A}(K)_{\ell}^{\vee}$ has no nontrivial pseudo-null submodule.
\end{abstract}

Keywords: Selmer groups, abelian varieties, function fields, pseudo-null modules. MSC (2010): 11R23 (primary), 11G35 (secondary).

\section{INTRODUCTION}

Let $G$ be a compact $\ell$-adic Lie group and $\Lambda(G)$ its associated Iwasawa algebra. A crucial theme in Iwasawa theory is the study of finitely generated $\Lambda(G)$-modules and their structure, up to "pseudo-isomorphism". When $G \simeq \mathbb{Z}_{\ell}^{d}$ for some integer $d \geqslant 1$, the structure theory for finitely generated $\Lambda(G)$-modules is well known (see, e.g., [B]). For a nonabelian $G$, which is the case we are interested in, studying this topic is possible thanks to an appropriate definition of the concept of "pseudo-null" for modules over $\Lambda(G)$ due to Venjakob (see [V]).

Let $F$ be a global function field of trascendence degree one over its constant field $\mathbb{F}_{q}$, where $q$ is a power of a fixed prime $p \in \mathbb{Z}$, and $K$ a Galois extension of $F$ unramified outside a finite set of primes $S$ and such that $G=\operatorname{Gal}(K / F)$ is an infinite $\ell$-adic Lie group with $\ell \neq p$. Let $A / F$ be an abelian variety: the structure of $\mathcal{S}:=\operatorname{Sel}_{A}(K)_{\ell}^{\vee}$ (the Pontrjagin dual of the Selmer group of $A$ over $K)$ as a $\Lambda(G)$-module has been extensively studied, for example, in [BL], [BL2] and $[\mathrm{T}]$ (see also the short survey in [BBL, Section 2] and the references there) for the abelian case, and in $[\mathrm{OT}],[\mathrm{W}]$ and $[\mathrm{BV}]$ for the noncommutative one (these results cover also the case $\ell=p$ ). In most cases $\mathcal{S}$ has been proved to be a finitely generated (sometimes torsion) $\Lambda(G)$-module and here we shall deal with the presence of nontrivial pseudo-null submodules in $\mathcal{S}$. For the number field setting and $K=F\left(A\left[\ell^{\infty}\right]\right)$, this issue was studied by Ochi and Venjakob ([OV, Theorem 5.1]) when $A$ is an elliptic curve, and by Ochi for a general abelian variety in $[\mathrm{O}]$ (see also $[\mathrm{HV}]$ and $[\mathrm{HO}]$ for analogous results and/or alternative proofs).

In Sections 2 and 3 we give a brief description of the objects we will work with and of the main tools we shall need, adapting some of the techniques of [OV] to our function field setting and to a general $\ell$-adic Lie extension (one of the main difference being the triviality of the image of the local Kummer maps).

In Section 4 we will prove the following

Theorem 1.1 (Theorem 4.1). Let $G=\operatorname{Gal}(K / F)$ be an $\ell$-adic Lie group without elements of order $\ell$ and of positive dimension $d \geqslant 3$. If $H^{2}\left(F_{S} / K, A\left[\ell^{\infty}\right]\right)=0$ and the map $\psi$ (induced by restriction)

$$
\operatorname{Sel}_{A}(K)_{\ell} \hookrightarrow H^{1}\left(F_{S} / K, A\left[\ell^{\infty}\right]\right) \stackrel{\psi}{\longrightarrow} \bigoplus_{S} \operatorname{Coind}_{G}^{G_{v}} H^{1}\left(K_{w}, A\right)\left[\ell^{\infty}\right]
$$

is surjective, then $\operatorname{Sel}_{A}(K)_{\ell}^{\vee}$ has no nontrivial pseudo-null submodule.

For the case $d=2$ we need more restrictive hypotheses, in particular we have the following 
Proposition 1.2 (Proposition 4.3). Let $G=\operatorname{Gal}(K / F)$ be an $\ell$-adic Lie group without elements of order $\ell$ and of dimension $d \geqslant 2$. If $H^{2}\left(F_{S} / K, A\left[\ell^{\infty}\right]\right)=0$ and $\operatorname{cd}_{\ell}\left(G_{v}\right)=2$ for any $v \in S$, then $\operatorname{Sel}_{A}(K)_{\ell}^{\vee}$ has no nontrivial pseudo-null submodule.

A few considerations and particular cases for the vanishing of $H^{2}\left(F_{S} / K, A\left[\ell^{\infty}\right]\right)$ are included at the end of Section 4.

Acknowledgements The authors thank F. Bars for valuable discussions and comments on earlier drafts of this paper.

\section{Setting AND Notations}

Here we fix notations and conventions that will be used through the paper.

2.1. Fields and extensions. Let $F$ be a global function field of trascendence degree one over its constant field $\mathbb{F}_{F}=\mathbb{F}_{q}$, where $q$ is a power of a fixed prime $p \in \mathbb{Z}$. We put $\bar{F}$ for an algebraic closure of $F$.

For any algebraic extension $L / F$, let $\mathfrak{M}_{L}$ be the set of places of $L$ : for any $v \in \mathfrak{M}_{L}$ we let $L_{v}$ be the completion of $L$ at $v$. Let $S$ be a finite nonempty subset of $\mathfrak{M}_{F}$ and let $F_{S}$ be the maximal Galois extension of $F$ unramified outside $S$ with $G_{S}(F):=\operatorname{Gal}\left(F_{S} / F\right)$. Put $\mathcal{O}_{L, S}$ as the ring of $S$-integers of $L$ and $\mathcal{O}_{S}^{\times}$as the units of $\mathcal{O}_{S}=\bigcup_{L \subset F_{S}} \mathcal{O}_{L, S}$. Finally, $C \ell_{S}(L)$ denotes the $S$-ideal class group of $\mathcal{O}_{L, S}$ : since $S$ is nonempty, $C \ell_{S}(L)$ is finite.

For any place $v \in \mathfrak{M}_{F}$ we choose (and fix) an embedding $\bar{F} \hookrightarrow \overline{F_{v}}$, in order to get a restriction $\operatorname{map} G_{F_{v}}:=\operatorname{Gal}\left(\overline{F_{v}} / F_{v}\right) \hookrightarrow G_{F}:=\operatorname{Gal}(\bar{F} / F)$.

We will deal with $\ell$-adic Lie extensions $K / F$, i.e., Galois extensions with Galois group an $\ell$-adic Lie group with $\ell \neq p$. We always assume that our extensions are unramified outside a finite set $S$ of primes of $\mathfrak{M}_{F}$.

In what follows $\operatorname{Gal}(K / F)$ is an $\ell$-adic Lie group without points of order $\ell$, then it has finite $\ell$ cohomological dimension, which is equal to its dimension as an $\ell$-adic Lie group ([Se, Corollaire (1) p. 413]).

2.2. Ext and duals. For any $\ell$-adic Lie group $G$ we denote by

$$
\Lambda(G)=\mathbb{Z}_{\ell}[[G]]:=\lim _{\overleftarrow{U}} \mathbb{Z}_{\ell}[G / U]
$$

the associated Iwasawa algebra (the limit is on the open normal subgroups of $G$ ). From Lazard's work (see [L]), we know that $\Lambda(G)$ is Noetherian and, if $G$ is pro- $\ell$ and has no elements of order $\ell$, then $\Lambda(G)$ is an integral domain.

For a $\Lambda(G)$-module $M$ we consider the extension groups

$$
\mathrm{E}^{i}(M):=\operatorname{Ext}_{\Lambda(G)}^{i}(M, \Lambda(G))
$$

for any integer $i$ and put $\mathrm{E}^{i}(M)=0$ for $i<0$ by convention.

Since in our applications $G$ comes from a Galois extension, we denote with $G_{v}$ the decomposition group of $v \in \mathfrak{M}_{F}$ for some prime $w \mid v, w \in \mathfrak{M}_{L}$, and we use the notation

$$
\mathrm{E}_{v}^{i}(M):=\operatorname{Ext}_{\Lambda\left(G_{v}\right)}^{i}\left(M, \Lambda\left(G_{v}\right)\right) .
$$

Let $H$ be a closed subgroup of $G$. For every $\Lambda(H)$-module $N$ we consider the $\Lambda(G)$-modules

$$
\operatorname{Coind}_{G}^{H}(N):=\operatorname{Map}_{\Lambda(H)}(\Lambda(G), N) \text { and } \operatorname{Ind}_{H}^{G}(N):=N \otimes_{\Lambda(H)} \Lambda(G){ }^{1} .
$$

\footnotetext{
${ }^{1}$ We use the notations of $[\mathrm{OV}]$, some texts, e.g. $[\mathrm{NSW}]$, switch the definitions of $\operatorname{Ind}_{G}^{H}(N)$ and $\operatorname{Coind}_{G}^{H}(N)$.
} 
For a $\Lambda(G)$-module $M$, we denote its Pontrjagin dual by $M^{\vee}:=\operatorname{Hom}_{\text {cont }}\left(M, \mathbb{Q}_{\ell} / \mathbb{Z}_{\ell}\right)$. In this

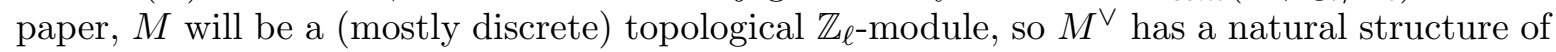
$\mathbb{Z}_{\ell \text {-module. }}$

If $M$ is a discrete $G_{S}(F)$-module, finitely generated over $\mathbb{Z}$ and with no $p$-torsion, in duality theorems we shall use also the dual $G_{S}(F)$-module of $M$, i.e.,

$$
M^{\prime}:=\operatorname{Hom}\left(M, \mathcal{O}_{S}^{\times}\right)(=\operatorname{Hom}(M, \boldsymbol{\mu}) \text { if } M \text { is finite }) .
$$

2.3. Selmer groups. Let $A$ be an abelian variety of dimension $g$ defined over $F$ : we denote by $A^{t}$ its dual abelian variety. For any positive integer $n$ we let $A[n]$ be the scheme of $n$-torsion points and, for any prime $\ell$, we put $A\left[\ell^{\infty}\right]:=\lim _{\rightarrow} A\left[\ell^{n}\right]$.

The local Kummer maps (for any $w \in \mathfrak{M}_{L}$ )

$$
\kappa_{w}: A\left(L_{w}\right) \otimes \mathbb{Q}_{\ell} / \mathbb{Z}_{\ell} \hookrightarrow \lim _{\vec{n}} H^{1}\left(L_{w}, A\left[\ell^{n}\right]\right):=H^{1}\left(L_{w}, A\left[\ell^{\infty}\right]\right)
$$

(arising from the cohomology of the exact sequence $A\left[\ell^{n}\right] \hookrightarrow A_{\ell^{n}}^{\rightarrow} A$ ) enable one to define the $\ell$-part of the Selmer group of $A$ over $L$ as

$$
\operatorname{Sel}_{A}(L)_{\ell}=\operatorname{Ker}\left\{H^{1}\left(L, A\left[\ell^{\infty}\right]\right) \rightarrow \prod_{w \in \mathfrak{M}_{L}} H^{1}\left(L_{w}, A\left[\ell^{\infty}\right]\right) / \operatorname{Im} \kappa_{w}\right\}
$$

(where the map is the product of the natural restrictions between cohomology groups).

For infinite extensions $\mathcal{L} / F$ the Selmer group $\operatorname{Sel}_{A}(\mathcal{L})_{\ell}$ is defined, as usual, via direct limits.

Since $\ell \neq p$, the $I m \kappa_{w}$ are trivial and, assuming that $S$ contains also all primes of bad reduction for $A$, we have the following equivalent

Definition 2.1. The $\ell$-part of the Selmer group of $A$ over $L$ is

$$
\operatorname{Sel}_{A}(L)_{\ell}=\operatorname{Ker}\left\{H^{1}\left(F_{S} / L, A\left[\ell^{\infty}\right]\left(F_{S}\right)\right) \rightarrow \bigoplus_{S} \operatorname{Coind}_{G}^{G_{v}} H^{1}\left(L_{w}, A\left[\ell^{\infty}\right]\right)\right\} .
$$

Letting $L$ vary through subextensions of $K / F$, the groups $\operatorname{Sel}_{A}(L)_{\ell}$ admit natural actions by $\mathbb{Z}_{\ell}$ (because of $A\left[\ell^{\infty}\right]$ ) and by $G=\operatorname{Gal}(K / F)$. Hence they are modules over the Iwasawa algebra $\Lambda(G)$.

\section{Homotopy THEORY AND PSEUdO-NULLITY}

We briefly recall the basic definitions for pseudo-null modules over a non-commutative Iwasawa algebra: a comprehensive reference is $[\mathrm{V}]$.

3.1. Pseudo-null $\Lambda(G)$-modules. Let $G$ be an $\ell$-adic Lie group without $\ell$-torsion, then $\Lambda(G)$ is an Auslander regular ring of finite global dimension $\mathfrak{d}=\operatorname{cd}_{\ell}(G)+1\left(\left[\mathrm{~V}\right.\right.$, Theorem 3.26], $\mathrm{cd}_{\ell}$ denotes the $\ell$-cohomological dimension).

For any finitely generated $\Lambda(G)$-module $M$, there is a canonical filtration

$$
T_{0}(M) \subseteq T_{1}(M) \subseteq \cdots \subseteq T_{\mathfrak{d}-1}(M) \subseteq T_{\mathfrak{d}}(M)=M .
$$

Definition 3.1. We say that a $\Lambda(G)$-module $M$ is pseudo-null if

$$
\delta(M):=\min \left\{i \mid T_{i}(M)=M\right\} \leqslant \mathfrak{d}-2 .
$$


The quantity $\delta(M)$, called the $\delta$-dimension of the $\Lambda(G)$-module $M$, is used along with the grade of $M$, that is

$$
j(M):=\min \left\{i \mid \mathrm{E}^{i}(M) \neq 0\right\} .
$$

As $j(M)+\delta(M)=\mathfrak{d}$ ([V, Proposition 3.5 (ii)]) we have that $M$ is a pseudo-null module if and only if $\mathrm{E}^{0}(M)=\mathrm{E}^{1}(M)=0$.

Since $\delta\left(T_{i}(M)\right) \leqslant i$ and every $T_{i}(M)$ is the maximal submodule of $M$ with $\delta$-dimension less or equal to $i$ ([V, Proposition 3.5 (vi) (a)]), only $T_{0}(M), \ldots, T_{\mathfrak{d}-2}(M)$ can be pseudo-null. If $T_{0}(M)=\cdots=T_{\mathfrak{d}-2}(M)=0, M$ does not have any nonzero pseudo-null submodule. This is the case when $\mathrm{E}^{i} \mathrm{E}^{i}(M)=0 \forall i \geqslant 2([\mathrm{~V}$, Proposition 3.5 (i) (c)]).

3.2. The powerful diagram and its consequences. In [OV, Lemma 4.5] Ochi and Venjakob generalized a result of Jannsen (see $[\mathrm{J}]$ ) which is very powerful in applications (they call it "powerful diagram"). We provide here the statements we shall need later: for the missing details of the proofs the reader can consult [NSW, Chapter V, Section 5] and/or [OV, Section 4] (those results hold in our setting as well because we work with the $\Lambda(G)$-module $A\left[\ell^{\infty}\right]$, with $\ell \neq p)$.

Replacing, if necessary, $F$ by a finite extension we can (and will) assume that $K$ is contained in the maximal pro- $\ell$ extension of $F_{\infty}:=F\left(A\left[\ell^{\infty}\right]\right)$ unramified outside $S$. Then we have the following

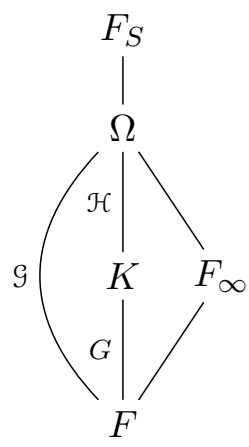

where $\Omega$ is the maximal pro- $\ell$ extension of $F_{\infty}$ contained in $F_{S}$. We put $\mathcal{G}=\operatorname{Gal}(\Omega / F)$, $\mathcal{H}=\operatorname{Gal}(\Omega / K)$ and $G=\operatorname{Gal}(K / F)$. The extension $F_{\infty} / F$ will be called the trivializing extension.

Tensoring the natural exact sequence $I(\mathcal{G}) \hookrightarrow \Lambda(\mathcal{G}) \rightarrow \mathbb{Z}_{\ell}$ with $A\left[\ell^{\infty}\right]^{\vee} \simeq \mathbb{Z}_{\ell}^{2 g}$, one gets

$$
I(\mathcal{G}) \otimes_{\mathbb{Z}_{\ell}} A\left[\ell^{\infty}\right]^{\vee} \hookrightarrow \Lambda(\mathcal{G}) \otimes_{\mathbb{Z}_{\ell}} A\left[\ell^{\infty}\right]^{\vee} \rightarrow A\left[\ell^{\infty}\right]^{\vee} .
$$

Since the mid term is projective ([OV, Lemma 4.2]), the previous sequence yields

$$
H_{1}\left(\mathcal{H}, A\left[\ell^{\infty}\right]^{\vee}\right) \hookrightarrow\left(I(\mathcal{G}) \otimes_{\mathbb{Z}_{\ell}} A\left[\ell^{\infty}\right]^{\vee}\right)_{\mathcal{H}} \rightarrow\left(\Lambda(\mathcal{G}) \otimes_{\mathbb{Z}_{\ell}} A\left[\ell^{\infty}\right]^{\vee}\right)_{\mathcal{H}} \rightarrow\left(A\left[\ell^{\infty}\right]^{\vee}\right)_{\mathcal{H}} .
$$

In order to shorten notations we put:

- $X=H_{1}\left(\mathcal{H}, A\left[\ell^{\infty}\right]^{\vee}\right)$

- $Y=\left(I(\mathcal{G}) \otimes_{\mathbb{Z}_{\ell}} A\left[\ell^{\infty}\right]^{\vee}\right)_{\mathcal{H}}$

- $J=K \operatorname{Ker}\left\{\left(\Lambda(\mathcal{G}) \otimes_{\mathbb{Z}_{\ell}} A\left[\ell^{\infty}\right]^{\vee}\right)_{\mathcal{H}} \rightarrow\left(A\left[\ell^{\infty}\right]^{\vee}\right)_{\mathcal{H}}\right\}$.

So the sequence (1) becomes

$$
X \hookrightarrow Y \rightarrow J .
$$

For our purpose it is useful to think of $X$ as $H^{1}\left(F_{S} / K, A\left[\ell^{\infty}\right]\right)^{\vee}$ (note that $H_{1}\left(\mathcal{H}, A\left[\ell^{\infty}\right]^{\vee}\right) \simeq$ $\left.H^{1}\left(\Omega / K, A\left[\ell^{\infty}\right]\right)^{\vee} \simeq H^{1}\left(F_{S} / K, A\left[\ell^{\infty}\right]\right)^{\vee}\right)$.

Let $\mathcal{F}(d)$ denote a free pro- $\ell$-group of rank $d=\operatorname{dim} \mathcal{G}$ and denote by $\mathcal{N}$ (resp. $\mathcal{R}$ ) the kernel of the natural map $\mathcal{F}(d) \rightarrow \mathcal{G}$ (resp. $\mathcal{F}(d) \rightarrow G$ ). For any profinite group $H$, we denote by 
$H^{a b}(\ell)$ the maximal pro- $\ell$-quotient of the maximal abelian quotient of $H$. With this notations the powerful diagram reads as

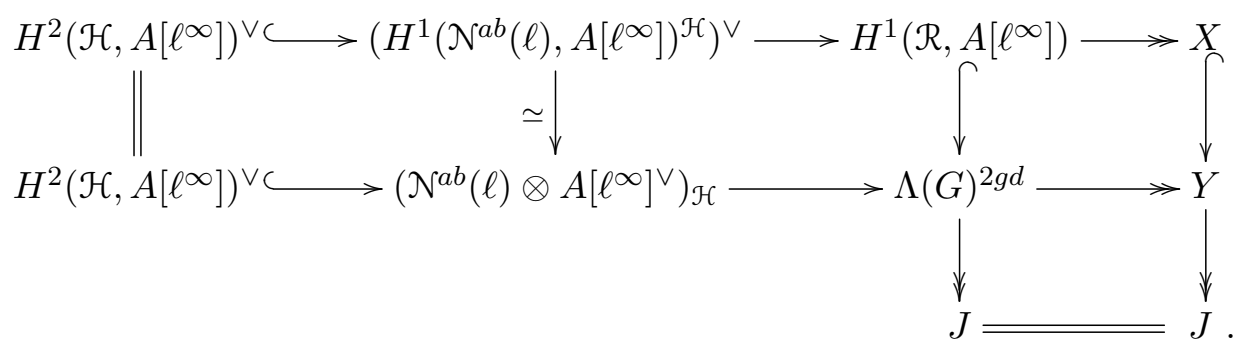

Moreover, since $c d_{\ell}(\mathcal{G}) \leqslant 2$ (just use [NSW, Theorem 8.3.17] and work as in [OV, Lemma 4.4 , (iv)]), the module $\mathcal{N}^{a b}(\ell) \otimes A\left[\ell^{\infty}\right]^{\vee}$ is free over $\Lambda(\mathcal{G})([\mathrm{OV}$, Lemma 4.2$])$, hence $\left(\mathcal{N}^{a b}(\ell) \otimes\right.$ $\left.A\left[\ell^{\infty}\right]^{\vee}\right)_{\mathcal{H}}$ is projective as a $\Lambda(\mathcal{G} / \mathcal{H})=\Lambda(G)$-module. Therefore, if $H^{2}\left(F_{S} / K, A\left[\ell^{\infty}\right]\right)=0$, the module $Y$ has projective dimension $\leqslant 1$. Whenever this is true the definition of $J$ provides the isomorphisms

$$
\mathrm{E}^{i}(X) \simeq \mathrm{E}^{i+1}(J) \quad \text { and } \quad E^{i}(J) \simeq \mathrm{E}^{i+1}\left(\left(A\left[\ell^{\infty}\right]^{\vee}\right)_{\mathcal{H}}\right) \quad \forall i \geqslant 2,
$$

which will be repeatedly used in our computations.

We shall need also a "localized" version of the sequence (2). For every $v \in S$ and a $w \in \mathfrak{M}_{K}$ dividing $v$, we define

$$
X_{v}=H^{1}\left(K_{w}, A\left[\ell^{\infty}\right]\right)^{\vee} \quad \text { and } \quad Y_{v}=\left(I\left(\mathcal{G}_{v}\right) \otimes_{\mathbb{Z}_{\ell}} A\left[\ell^{\infty}\right]^{\vee}\right)_{\mathcal{H}_{v}}
$$

(with $\mathcal{G}_{v}$ the decomposition groups of $v$ in $\mathcal{G}$ and $\mathcal{H}_{v}=\mathcal{H} \cap \mathcal{G}_{v}$ ). The exact sequence

$$
X_{v} \hookrightarrow Y_{v} \rightarrow J_{v}
$$

fits into the localized version of diagram (3). If $K_{w}$ is still a local field, then Tate local duality ([NSW, Theorem 7.2.6]) yields

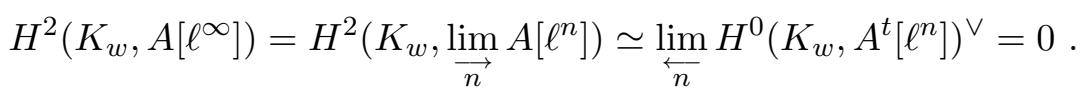

If $K_{w}$ is not local, then $\ell^{\infty}$ divides the degree of the extension $K_{w} / F_{v}$ and $H^{2}\left(K_{w}, A\left[\ell^{\infty}\right]\right)=0$ by [NSW, Theorem 7.1.8 (i)]. Therefore $Y_{v}$ always has projective dimension $\leqslant 1$ and

$$
\mathrm{E}^{i}\left(X_{v}\right) \simeq \mathrm{E}^{i+1}\left(J_{v}\right) \simeq \mathrm{E}^{i+2}\left(\left(A\left[\ell^{\infty}\right]^{\vee}\right)_{\mathcal{H}_{v}}\right) \quad \forall i \geqslant 2 .
$$

We note that, since $\ell \neq p$, the image of the local Kummer maps is always 0 , hence

$$
X_{v}=H^{1}\left(K_{w}, A\left[\ell^{\infty}\right]\right)^{\vee}=\left(H^{1}\left(K_{w}, A\left[\ell^{\infty}\right]\right) / \operatorname{Im} \kappa_{w}\right)^{\vee} \simeq H^{1}\left(K_{w}, A\right)\left[\ell^{\infty}\right]^{\vee} .
$$

Then Definition 2.1 for $L=K$ can be written as

$$
\operatorname{Sel}_{A}(K)_{\ell}=\operatorname{Ker}\left\{\psi: X^{\vee} \longrightarrow \bigoplus_{S} \operatorname{Coind}_{G}^{G_{v}} X_{v}^{\vee}\right\}
$$

and, dualizing, we get a map

$$
\psi^{\vee}: \bigoplus_{S} \operatorname{Ind}_{G_{v}}^{G} X_{v} \longrightarrow X
$$

whose cokernel is exactly $\mathcal{S}:=\operatorname{Sel}_{A}(K)_{\ell}^{\vee}$.

The following result will be fundamental for our computations.

Theorem 3.2 (U. Jannsen). Let $G$ be an $\ell$-adic Lie group without elements of order $\ell$ and of dimension $d$. Let $M$ be a $\Lambda(G)$-module which is finitely generated as $\mathbb{Z}_{\ell}$-module. Then $\mathrm{E}^{i}(M)$ is a finitely generated $\mathbb{Z}_{\ell}$-module and, in particular,

1. if $M$ is $\mathbb{Z}_{\ell}$-free, then $E^{i}(M)=0$ for any $i \neq d$ and $E^{d}(M)$ is free; 
2. if $M$ is finite, then $E^{i}(M)=0$ for any $i \neq d+1$ and $E^{d+1}(M)$ is finite.

Proof. See [J, Corollary 2.6].

Corollary 3.3. With notations as above:

1. if $H^{2}\left(F_{S} / K, A\left[\ell^{\infty}\right]\right)=0$, then, for $i \geqslant 2$,

$$
\mathrm{E}^{i}(X) \text { is } \begin{cases}\text { finite } & \text { if } i=d-1 \\ \text { free } & \text { if } i=d-2 \\ 0 & \text { otherwise }\end{cases}
$$

2. $\mathrm{E}_{v}^{i} \mathrm{E}_{v}^{i-1}\left(X_{v}\right)=0$ for $i \geqslant 3$.

Proof. 1. The hypothesis yields the isomorphism $\mathrm{E}^{i}(X) \simeq E^{i+2}\left(\left(A\left[\ell^{\infty}\right]^{\vee}\right)_{\mathcal{H}}\right)$. Since

$$
\left(A\left[\ell^{\infty}\right]^{\vee}\right)_{\mathcal{H}} \simeq\left(A\left[\ell^{\infty}\right]^{\mathcal{H}}\right)^{\vee}=A\left[\ell^{\infty}\right](K)^{\vee} \simeq \mathbb{Z}_{\ell}^{r} \oplus \Delta
$$

(with $0 \leqslant r \leqslant 2 g$ and $\Delta$ a finite group) and $\mathrm{E}^{i}\left(\mathbb{Z}_{\ell}^{r} \oplus \Delta\right)=\mathrm{E}^{i}\left(\mathbb{Z}_{\ell}^{r}\right) \oplus \mathrm{E}^{i}(\Delta)$, the claim follows from Theorem 3.2.

2. Use Theorem 3.2 and the isomorphism in (6).

Lemma 3.4. If $H^{2}\left(F_{S} / K, A\left[\ell^{\infty}\right]\right)=0$, then there is the following commutative diagram

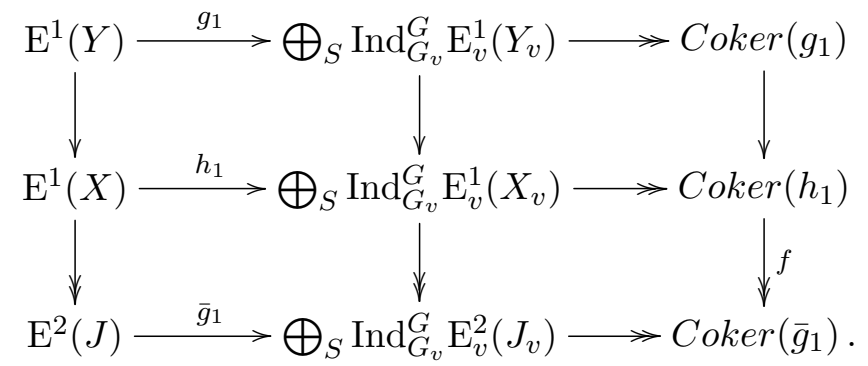

Proof. The inclusions $\mathcal{G}_{v} \subseteq \mathcal{G}$ and $\mathcal{H}_{v} \subseteq \mathcal{H}$ induce the maps

$$
\left(I\left(\mathcal{G}_{v}\right) \otimes_{\mathbb{Z}_{\ell}} A\left[\ell^{\infty}\right]^{\vee}\right)_{\mathcal{H}_{v}} \rightarrow\left(I(\mathcal{G}) \otimes_{\mathbb{Z}_{\ell}} A\left[\ell^{\infty}\right]^{\vee}\right)_{\mathcal{H}_{v}} \rightarrow\left(I(\mathcal{G}) \otimes_{\mathbb{Z}_{\ell}} A\left[\ell^{\infty}\right]^{\vee}\right)_{\mathcal{H}}
$$

We have a homomorphism of $\Lambda(G)$-modules $g: \bigoplus_{S} \operatorname{Ind}_{G_{v}}^{G} Y_{v} \rightarrow Y$ which, restricted to the $X_{v}$ 's, provides the map $h: \bigoplus_{S} \operatorname{Ind}_{G_{v}}^{G} X_{v} \rightarrow X$. So we have the following situation

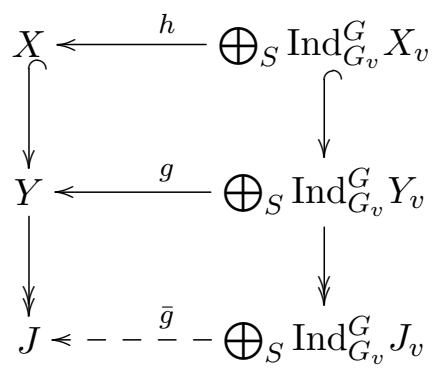

where $\bar{g}$ is induced by $g$ and the diagram is obviously commutative.

Since $Y$ and the $Y_{v}$ 's have projective dimension $\leqslant 1$ (i.e., $\mathrm{E}^{2}(Y)=\mathrm{E}^{2}\left(Y_{v}\right)=0$ ), the lemma follows by taking Ext in diagram (7) and recalling that, for any $i \geqslant 0, \mathrm{E}_{v}^{i}\left(\operatorname{Ind}_{G_{v}}^{G}\left(X_{v}\right)\right)=$ $\operatorname{Ind}_{G_{v}}^{G} \mathrm{E}_{v}^{i}\left(X_{v}\right)$ (see [OV, Lemma 5.5]).

In the next subsection we are going to describe the structure of $\operatorname{Coker}\left(g_{1}\right)$. 
3.3. Homotopy theory and Coker $\left(g_{1}\right)$. For every finitely generated $\Lambda(G)$-module $M$ choose a presentation $P_{1} \rightarrow P_{0} \rightarrow M \rightarrow 0$ of $M$ by projectives and define the transpose functor $D M$ by the exactness of the sequence

$$
0 \rightarrow \mathrm{E}^{0}(M) \rightarrow \mathrm{E}^{0}\left(P_{0}\right) \rightarrow \mathrm{E}^{0}\left(P_{1}\right) \rightarrow D M \rightarrow 0 .
$$

Then it can be shown that the functor $D$ is well-defined and one has $D^{2}=I d$ (see $[\mathrm{J}]$ ).

Definition 3.5. Let $L$ be an extension of $F$ contained in $F_{S}$. Then we define

$$
Z(L):=H^{0}\left(F_{S} / L, \lim _{\vec{m}} D_{2}\left(A\left[\ell^{m}\right]\right)\right)^{\vee}
$$

where

$$
D_{2}\left(A\left[\ell^{m}\right]\right)=\lim _{F \subset \overrightarrow{E \subset} F_{S}}\left(H^{2}\left(F_{S} / E, A\left[\ell^{m}\right]\right)\right)^{\vee}
$$

and the limit in $\underset{\vec{m}}{\lim _{\rightarrow}} D_{2}\left(A\left[\ell^{m}\right]\right)$ is taken with respect to the $\ell$-power map $A\left[\ell^{m+1}\right] \stackrel{\ell}{\rightarrow} A\left[\ell^{m}\right]$.

In the same way we define $Z(L)$ for any Galois extension $L$ of $F_{v}$.

An alternative description of the module $Z$ is provided by the following

Lemma 3.6. Let $K$ be a fixed extension of $F$ contained in $F_{S}$ and $K_{w}$ its completion for some $w \mid v \in S$. Then

$$
Z(K) \simeq \lim _{F \subseteq \overleftarrow{L} \subseteq K} H^{2}\left(F_{S} / L, T_{\ell}(A)\right) \quad \text { and } \quad Z\left(K_{w}\right) \simeq \lim _{F_{v} \subseteq \overleftarrow{L \subseteq} K_{w}} H^{2}\left(L, T_{\ell}(A)\right)
$$

Proof. Global case. For any global field $L$, let

$$
\amalg^{i}\left(F_{S} / L, A\left[\ell^{\infty}\right]\right):=\operatorname{Ker}\left\{H^{i}\left(F_{S} / L, A\left[\ell^{\infty}\right]\right) \rightarrow \bigoplus_{S} H^{i}\left(L_{w}, A\left[\ell^{\infty}\right]\right)\right\} .
$$

We have already seen that $H^{2}\left(L_{w}, A\left[\ell^{\infty}\right]\right)=0$, hence $H^{2}\left(F_{S} / L, A\left[\ell^{\infty}\right]\right)=\amalg^{2}\left(F_{S} / L, A\left[\ell^{\infty}\right]\right)$. Using the pairing of [M, Ch. I, Proposition 6.9], we get

$$
\begin{aligned}
Z(K) & =H^{0}\left(F_{S} / K, \lim _{\vec{m}} \lim _{F \subseteq \overrightarrow{L \subseteq} F_{S}} \amalg^{2}\left(F_{S} / L, A\left[\ell^{m}\right]\right)^{\vee}\right)^{\vee} \\
& =H^{0}\left(F_{S} / K, \lim _{\vec{m}} \lim _{F \subseteq \overrightarrow{L \subseteq} F_{S}} \amalg^{0}\left(F_{S} / L, A^{t}\left[\ell^{m}\right]\right)\right)^{\vee} \\
& =\left(\lim _{\vec{m}} \lim _{F \subseteq \overrightarrow{L \subseteq} F_{S}} \amalg^{0}\left(F_{S} / L, A^{t}\left[\ell^{m}\right]\right)^{\operatorname{Gal}\left(F_{S} / K\right)}\right)^{\vee} \\
& \left.=\lim _{\vec{m}} \lim _{F \subseteq \overrightarrow{L \subseteq K}} \amalg^{0}\left(F_{S} / L, A^{t}\left[\ell^{m}\right]\right)\right)^{\vee} \\
& =\lim _{\overleftarrow{m}} \lim _{F \subseteq L \subseteq K}\left(H^{2}\left(F_{S} / L, A\left[\ell^{m}\right]\right)^{\vee}\right)^{\vee} \\
& =\lim _{F \subseteq \leftarrow \subseteq K} H^{2}\left(F_{S} / L, T_{\ell}(A)\right) .
\end{aligned}
$$

Local case. The proof is similar (using Tate local duality).

We recall that our group $G$ has no elements of order $\ell$, hence $\Lambda(G)$ is a domain. Moreover for any open subgroup $U$ of $G$ we have that (see [J, Lemma 2.3])

$$
\mathrm{E}^{i}(U) \simeq \mathrm{E}^{i}(G) \quad \forall i \in \mathbb{Z}
$$

is an isomorphism of $\Lambda(U)$-modules. An $\ell$-adic Lie group $G$ always contains an open pro- $\ell$ subgroup ([DdSMS, Corollary 8.34]), so, in order to use properly the usual definitions of "torsion submodule" and "rank" for a finitely generated $\Lambda(G)$-module, with no loss of generality, 
we will assume that $G$ is pro- $\ell$.

Proposition 3.7. Let $M$ be a finitely generated $\Lambda(G)$-module. Then $\mathrm{E}^{i}(M)$ is a finitely generated torsion $\Lambda(G)$-module for any $i \geqslant 1$.

Proof. Take a finite presentation $P_{1} \rightarrow P_{0} \rightarrow M \rightarrow 0$ with finitely generated and projective $\Lambda(G)$-modules $P_{1}$ and $P_{0}$, and the consequent exact sequence

$$
0 \rightarrow R_{1} \rightarrow P_{0} \rightarrow M \rightarrow 0
$$

for a suitable submodule $R_{1}$ of $P_{1}$. Since $M$ and $\operatorname{Hom}_{\Lambda(G)}(M, \Lambda(G))$ have the same $\Lambda(G)$-rank, computing ranks in the sequence coming from (8)

$$
\begin{aligned}
\operatorname{Hom}_{\Lambda(G)}(M, \Lambda(G)) \hookrightarrow \operatorname{Hom}_{\Lambda(G)}\left(P_{0}, \Lambda(G)\right) \rightarrow \operatorname{Hom}_{\Lambda(G)}\left(R_{1}, \Lambda(G)\right) \rightarrow \mathrm{E}^{1}(M) \rightarrow \\
\quad \rightarrow 0 \rightarrow \mathrm{E}^{1}\left(R_{1}\right) \rightarrow \mathrm{E}^{2}(M) \rightarrow 0 \rightarrow \cdots \rightarrow 0 \rightarrow \mathrm{E}^{i-1}\left(R_{1}\right) \rightarrow \mathrm{E}^{i}(M) \rightarrow 0 \rightarrow \cdots
\end{aligned}
$$

one finds $\operatorname{rank}_{\Lambda(G)}\left(\mathrm{E}^{1}(M)\right)=0$ for any finitely generated $\Lambda(G)$-module $M$. Therefore $\mathrm{E}^{1}\left(R_{1}\right)$ is torsion, which yields $\mathrm{E}^{2}(M) \simeq \mathrm{E}^{1}\left(R_{1}\right)$ is torsion. Iterating $\mathrm{E}^{i}(M) \simeq \mathrm{E}^{i-1}\left(R_{1}\right)$ is $\Lambda(G)$ torsion $\forall i \geqslant 2$.

Lemma 3.8. Let $F_{n}$ be subfields of $K$ such that $\operatorname{Gal}(K / F)=\underset{\leftarrow}{\lim _{n}} \operatorname{Gal}\left(F_{n} / F\right)$. Then

$$
H_{I w}^{2}\left(K_{w}, T_{\ell}(A)\right):=\lim _{\overleftarrow{n, m}} H^{2}\left(F_{v_{n}}, A\left[\ell^{m}\right]\right)
$$

is a torsion $\Lambda\left(G_{v}\right)$-module. If $H^{2}\left(F_{S} / K, A\left[\ell^{\infty}\right]\right)=0$, then

$$
H_{I w}^{2}\left(K, T_{\ell}(A)\right):=\lim _{\overleftarrow{n, m}} H^{2}\left(F_{S} / F_{n}, A\left[\ell^{m}\right]\right)
$$

is a $\Lambda(G)$-torsion as well.

Proof. The proofs are identical so we only show the second statement. From the spectral sequence

$$
\mathrm{E}_{2}^{p, q}=E^{p}\left(H^{q}\left(F_{S} / K, A\left[\ell^{\infty}\right]\right)^{\vee}\right) \Longrightarrow H_{I w}^{p+q}\left(K, T_{\ell}(A)\right)
$$

due to Jannsen (see [J1]), we have a filtration for $H_{I w}^{2}\left(K, T_{\ell}(A)\right)$

$$
0=H_{3}^{2} \subseteq H_{2}^{2} \subseteq H_{1}^{2} \subseteq H_{0}^{2}=H_{I w}^{2}\left(K, T_{\ell}(A)\right),
$$

which provides the following sequences:

$$
\begin{aligned}
\mathrm{E}^{0}\left(H^{1}\left(F_{S} / K, A\left[\ell^{\infty}\right]\right)^{\vee}\right) & \rightarrow \mathrm{E}^{2}\left(H^{0}\left(F_{S} / K, A\left[\ell^{\infty}\right]\right)^{\vee}\right) \rightarrow H_{1}^{2} \\
& \rightarrow \mathrm{E}^{1}\left(H^{1}\left(F_{S} / K, A\left[\ell^{\infty}\right]\right)^{\vee}\right) \rightarrow \mathrm{E}^{3}\left(H^{0}\left(F_{S} / K, A\left[\ell^{\infty}\right]\right)^{\vee}\right)
\end{aligned}
$$

and

$$
H_{1}^{2} \hookrightarrow H_{I w}^{2}\left(K, T_{\ell}(A)\right) \rightarrow \mathrm{E}_{\infty}^{0,2} .
$$

By hypothesis $\mathrm{E}_{\infty}^{0,2} \simeq \mathrm{E}_{2}^{0,2}=0$, so $H_{1}^{2} \simeq H_{I w}^{2}\left(K, T_{\ell}(A)\right)$.

Since $H^{i}\left(F_{S} / K, A\left[\ell^{\infty}\right]\right)^{\vee}$ is a finitely generated $\Lambda(G)$-module for $i \in\{0,1\}$ (for $i=1$ just look at $X$ in diagram (3)), Proposition 3.7 yields that the groups $\mathrm{E}^{2}\left(H^{0}\left(F_{S} / K, A\left[\ell^{\infty}\right]\right)^{\vee}\right)$ and $\mathrm{E}^{1}\left(H^{1}\left(F_{S} / K, A\left[\ell^{\infty}\right]\right)^{\vee}\right)$ are $\Lambda(G)$-torsion. Hence $H_{1}^{2}$ is torsion as well.

Lemma 3.9. With notations and hypotheses as in Lemma 3.4, Coker $\left(g_{1}\right)$ is finitely generated as $\mathbb{Z}_{\ell}$-module. 
Proof. Lemma 3.6 yields $Z(K)=H_{I w}^{2}\left(K, T_{\ell}(A)\right)$ so, using [OV, Proposition 4.10], one has $D H_{I w}^{2}\left(K, T_{\ell}(A)\right) \simeq Y$. Therefore $\mathrm{E}^{1}\left(D H_{I w}^{2}\left(K, T_{\ell}(A)\right)\right) \simeq \mathrm{E}^{1}(Y)$. Since $H_{I w}^{2}\left(K, T_{\ell}(A)\right)$ is a $\Lambda(G)$-torsion module, [OV, Lemma 3.1] implies $\mathrm{E}^{1}\left(D H_{I w}^{2}\left(K, T_{\ell}(A)\right) \simeq H_{I w}^{2}\left(K, T_{\ell}(A)\right)\right.$, i.e.,

$$
H_{I w}^{2}\left(K, T_{\ell}(A)\right) \simeq \mathrm{E}^{1}(Y)
$$

(the same holds for the "local" modules). The map $g_{1}$ of Lemma 3.4 then reads as

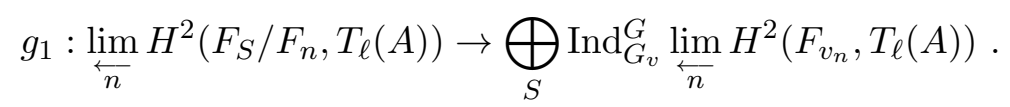

The claim follows from the Poitou-Tate sequence (see [NSW, 8.6.10 p. 488]), since

$$
\operatorname{Coker}\left(g_{1}\right) \simeq \lim _{\overleftarrow{n, m}} H^{0}\left(F_{S} / F_{n},\left(A\left[\ell^{m}\right]\right)^{\prime}\right)
$$

\section{MAIN TheOREM}

We are now ready to prove the following

Theorem 4.1. Let $G=\operatorname{Gal}(K / F)$ be an $\ell$-adic Lie group without elements of order $\ell$ and of positive dimension $d \geqslant 3$. If $H^{2}\left(F_{S} / K, A\left[\ell^{\infty}\right]\right)=0$ and the map $\psi$ in the sequence

$$
\operatorname{Sel}_{A}(K)_{\ell} \hookrightarrow H^{1}\left(F_{S} / K, A\left[\ell^{\infty}\right]\right) \stackrel{\psi}{\longrightarrow} \bigoplus_{S} \operatorname{Coind}_{G}^{G_{v}} H^{1}\left(K_{w}, A\right)\left[\ell^{\infty}\right]
$$

is surjective, then $\mathcal{S}:=\operatorname{Sel}_{A}(K)_{\ell}^{\vee}$ has no nontrivial pseudo-null submodule.

Proof. We need to prove that

$$
\mathrm{E}^{i} \mathrm{E}^{i}(\mathcal{S})=0 \quad \forall i \geqslant 2
$$

and we consider two cases.

Case $i=2$. Let $\mathcal{D}:=\bar{g}_{1}\left(\mathrm{E}^{2}(J)\right)$. Then

$$
\operatorname{Coker}\left(\bar{g}_{1}\right)=\bigoplus_{S} \operatorname{Ind}_{G_{v}}^{G} \mathrm{E}^{2}\left(J_{v}\right) / \mathcal{D}
$$

Observe that $\mathcal{D} \simeq \bar{g}_{1}\left(\mathrm{E}^{3}\left(A\left[\ell^{\infty}\right]_{\mathcal{H}}^{\vee}\right)\right)$ is a finitely generated $\mathbb{Z}_{\ell}$-module (it is zero if $d \neq 3$ and

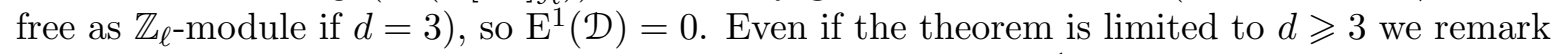
here that, for $d=2, \mathcal{D}$ is finite and, for $d=1, \mathcal{D}=0$ : hence $\mathrm{E}^{1}(\mathcal{D})=0$ in any case.

Moreover

$$
\begin{aligned}
\mathrm{E}^{2}\left(\bigoplus_{S} \operatorname{Ind}_{G_{v}}^{G} \mathrm{E}^{2}\left(J_{v}\right)\right) & =\mathrm{E}^{2}\left(\bigoplus_{S} \operatorname{Ind}_{G_{v}}^{G} \mathrm{E}^{3}\left(A\left[\ell^{\infty}\right]_{\mathcal{H}_{v}}^{\vee}\right)\right) \\
& =\bigoplus_{S} \operatorname{Ind}_{G_{v}}^{G} \mathrm{E}^{2} \mathrm{E}^{3}\left(A\left[\ell^{\infty}\right]_{\mathcal{H}_{v}}^{\vee}\right)=0,
\end{aligned}
$$

so, taking Ext in the sequence,

$$
\mathcal{D} \hookrightarrow \bigoplus_{S} \operatorname{Ind}_{G_{v}}^{G} \mathrm{E}^{2}\left(J_{v}\right) \rightarrow \bigoplus_{S} \operatorname{Ind}_{G_{v}}^{G} \mathrm{E}^{2}\left(J_{v}\right) / \mathcal{D}
$$

one finds

$$
\mathrm{E}^{1}(\mathcal{D}) \rightarrow \mathrm{E}^{2}\left(\bigoplus_{S} \operatorname{Ind}_{G_{v}}^{G} \mathrm{E}^{2}\left(J_{v}\right) / \mathcal{D}\right) \rightarrow \mathrm{E}^{2}\left(\bigoplus_{S} \operatorname{Ind}_{G_{v}}^{G} \mathrm{E}^{2}\left(J_{v}\right)\right)
$$

Therefore

$$
\mathrm{E}^{2}\left(\bigoplus_{S} \operatorname{Ind}_{G_{v}}^{G} \mathrm{E}^{2}\left(J_{v}\right) / \mathcal{D}\right)=0
$$


Recall the sequences

$$
\begin{gathered}
\bigoplus_{S} \operatorname{Ind}_{G_{v}}^{G} X_{v} \hookrightarrow X \rightarrow \mathcal{S} \\
\operatorname{Ker}(f) \hookrightarrow \operatorname{Coker}\left(h_{1}\right) \rightarrow \operatorname{Coker}\left(\bar{g}_{1}\right)
\end{gathered}
$$

provided (respectively) by the hypothesis on $\psi$ and by Lemma 3.4. Take Ext on (13) to get

$$
\mathrm{E}^{1}(X) \stackrel{h_{1}}{\longrightarrow} \mathrm{E}^{1}\left(\bigoplus_{S} \operatorname{Ind}_{G_{v}}^{G} X_{v}\right) \rightarrow \mathrm{E}^{2}(\mathcal{S}) \rightarrow \mathrm{E}^{2}(X) .
$$

If $d \geqslant 5$, then $\mathrm{E}^{2}(X) \simeq \mathrm{E}^{3}(J) \simeq \mathrm{E}^{4}\left(A\left[\ell^{\infty}\right]_{\mathcal{H}}^{\vee}\right)=0$. When this is the case Coker $\left(h_{1}\right) \simeq \mathrm{E}^{2}(\mathcal{S})$ and sequence (14) becomes

$$
\operatorname{Ker}(f) \hookrightarrow \mathrm{E}^{2}(\mathcal{S}) \rightarrow \bigoplus_{S} \operatorname{Ind}_{G_{v}}^{G} \mathrm{E}^{2}\left(J_{v}\right) / \mathcal{D} .
$$

By Lemma 3.9, $\operatorname{Ker}(f)$ is a finitely generated $\mathbb{Z}_{\ell}$-module. Taking Ext, one has

$$
\mathrm{E}^{2}\left(\bigoplus_{S} \operatorname{Ind}_{G_{v}}^{G} \mathrm{E}^{2}\left(J_{v}\right) / \mathcal{D}\right) \rightarrow \mathrm{E}^{2} \mathrm{E}^{2}(\mathcal{S}) \rightarrow \mathrm{E}^{2}(\operatorname{Ker}(f)),
$$

where the first and third term are trivial, so $\mathrm{E}^{2} \mathrm{E}^{2}(\mathcal{S})=0$ as well.

We are left with $d=3,4$. We know that $\mathrm{E}^{4}\left(A\left[\ell^{\infty}\right]_{\mathcal{H}}^{\vee}\right)=\mathrm{E}^{2}(X)$ is free over $\mathbb{Z}_{\ell}$ if $d=4$ or finite if $d=3$ (again we remark it is 0 if $d=1,2$ ). Anyway $\mathrm{E}^{2} \mathrm{E}^{2}(X)=0$ in all cases. From the sequence

$$
\operatorname{Coker}\left(h_{1}\right) \hookrightarrow \mathrm{E}^{2}(\mathcal{S}) \stackrel{\eta}{\longrightarrow} \mathrm{E}^{2}(X)
$$

one writes

$$
\operatorname{Coker}\left(h_{1}\right) \hookrightarrow \mathrm{E}^{2}(\mathcal{S}) \rightarrow \operatorname{Im}(\eta)
$$

where $\operatorname{Im}(\eta)$ is free over $\mathbb{Z}_{\ell}$ if $d=4$ or finite if $d=3$.

Taking Ext in (14) one has

$$
\mathrm{E}^{2}\left(\operatorname{Coker}\left(\bar{g}_{1}\right)\right) \rightarrow \mathrm{E}^{2}\left(\operatorname{Coker}\left(h_{1}\right)\right) \rightarrow \mathrm{E}^{2}(\operatorname{Ker}(f))
$$

with the first (see equation (12)) and third term equal to zero, so $\mathrm{E}^{2}\left(\operatorname{Coker}\left(h_{1}\right)\right)=0$. This fact in sequence (15) implies

$$
0=\mathrm{E}^{2}(\operatorname{Im}(\eta)) \rightarrow \mathrm{E}^{2} \mathrm{E}^{2}(\mathcal{S}) \rightarrow \mathrm{E}^{2}\left(\text { Coker }\left(h_{1}\right)\right)=0,
$$

so $\mathrm{E}^{2} \mathrm{E}^{2}(\mathcal{S})=0$.

Case $i \geqslant 3$. From sequence (13) we get the following

$$
\mathrm{E}^{i+1}\left(A\left[\ell^{\infty}\right]_{\mathcal{H}}^{\vee}\right) \simeq \mathrm{E}^{i-1}(X) \rightarrow \bigoplus_{S} \operatorname{Ind}_{G_{v}}^{G} \mathrm{E}_{v}^{i-1}\left(X_{v}\right) \rightarrow \mathrm{E}^{i}(\mathcal{S}) \rightarrow \mathrm{E}^{i}(X) \simeq \mathrm{E}^{i+2}\left(A\left[\ell^{\infty}\right]_{\mathcal{H}}^{\vee}\right)
$$

We have four cases, depending on whether $\mathrm{E}^{i-1}(X)$ and $\mathrm{E}^{i}(X)$ are trivial or not.

Case 1. Assume $\mathrm{E}^{i-1}(X)=\mathrm{E}^{i}(X)=0$.

From (16) we obtain the isomorphism

$$
\bigoplus_{S} \operatorname{Ind}_{G_{v}}^{G} \mathrm{E}_{v}^{i-1}\left(X_{v}\right) \simeq \mathrm{E}^{i}(\mathcal{S})
$$

so

$$
\bigoplus_{S} \operatorname{Ind}_{G_{v}}^{G} \mathrm{E}_{v}^{i} \mathrm{E}_{v}^{i-1}\left(X_{v}\right) \simeq \mathrm{E}^{i} \mathrm{E}^{i}(\mathcal{S})=0
$$

thanks to Corollary 3.3 part 2. We remark that this is the only case to consider when $d=1,2$. 
Case 2. Assume $\mathrm{E}^{i-1}(X)=0$ and $\mathrm{E}^{i}(X) \neq 0$.

This happens when $i=d-2$ or $i=d-1$ and $A\left[\ell^{\infty}\right]_{\mathcal{H}}^{\vee}$ is finite. From (16) we have

$$
\begin{gathered}
\bigoplus_{S} \operatorname{Ind}_{G_{v}}^{G} \mathrm{E}_{v}^{d-3} \hookrightarrow \mathrm{E}^{d-2}(\mathcal{S}) \rightarrow N \\
\left(\text { resp. } \bigoplus_{S} \operatorname{Ind}_{G_{v}}^{G} \mathrm{E}_{v}^{d-2} \hookrightarrow \mathrm{E}^{d-1}(\mathcal{S}) \rightarrow N\right)
\end{gathered}
$$

where $N$ is a submodule of the free module $\mathrm{E}^{d-2}(X)$ (resp. of the finite module $\left.\mathrm{E}^{d-1}(X)\right)$. Therefore $\mathrm{E}^{d-2}(N)=0$ (resp. $\mathrm{E}^{d-1}(N)=0$ ) and, moreover, $\mathrm{E}_{v}^{d-2} \mathrm{E}_{v}^{d-3}\left(X_{v}\right)=0$ (resp. $\mathrm{E}_{v}^{d-1} \mathrm{E}_{v}^{d-2}\left(X_{v}\right)=0$ ) by Corollary 3.3 part 2 . Hence $\mathrm{E}^{d-2} \mathrm{E}^{d-2}(\mathcal{S})=0\left(\right.$ resp. $\left.\mathrm{E}^{d-1} \mathrm{E}^{d-1}(\mathcal{S})=0\right)$. Case 3. Assume $\mathrm{E}^{i-1}(X) \neq 0$ and $\mathrm{E}^{i}(X)=0$.

This happens when $i=d$ or $i=d-1$ and $A\left[\ell^{\infty}\right]_{\mathcal{H}}^{\vee}$ is free. The sequence (16) gives

$$
\begin{gathered}
N \hookrightarrow \bigoplus_{S} \operatorname{Ind}_{G_{v}}^{G} \mathrm{E}_{v}^{d-1}\left(X_{v}\right) \rightarrow \mathrm{E}^{d}(\mathcal{S}) \\
\left(\operatorname{resp.} \quad N \hookrightarrow \bigoplus_{S} \operatorname{Ind}_{G_{v}}^{G} \mathrm{E}_{v}^{d-2}\left(X_{v}\right) \rightarrow \mathrm{E}^{d-1}(\mathcal{S})\right)
\end{gathered}
$$

where now $N$ is a quotient of the finite module $\mathrm{E}^{d-1}(X)$ (resp. of the free module $\left.\mathrm{E}^{d-2}(X)\right)$. Then $\mathrm{E}^{d}(N)=0\left(\right.$ resp. $\left.\mathrm{E}^{d-1}(N)=0\right)$ and

$$
\begin{gathered}
\bigoplus_{S} \operatorname{Ind}_{G_{v}}^{G} \mathrm{E}_{v}^{d} \mathrm{E}_{v}^{d-1}\left(X_{v}\right) \simeq \mathrm{E}^{d} \mathrm{E}^{d}(\mathcal{S})=0 \\
\left(\text { resp. } \quad \bigoplus_{S} \operatorname{Ind}_{G_{v}}^{G} \mathrm{E}_{v}^{d-1} \mathrm{E}_{v}^{d-2}\left(X_{v}\right) \simeq \mathrm{E}^{d-1} \mathrm{E}^{d-1}(\mathcal{S})=0\right) .
\end{gathered}
$$

Case 4. Assume $\mathrm{E}^{i-1}(X) \neq 0$ and $\mathrm{E}^{i}(X) \neq 0$.

This happens when $i=d-1$ and $A\left[\ell^{\infty}\right]_{\mathcal{H}}^{\vee}$ has nontrivial rank and torsion. From sequence (16) we have

$$
\mathrm{E}^{d-2}(X) \rightarrow \bigoplus_{S} \operatorname{Ind}_{G_{v}}^{G} \mathrm{E}_{v}^{d-2}\left(X_{v}\right) \rightarrow \mathrm{E}^{d-1}(\mathcal{S}) \rightarrow \mathrm{E}^{d-1}(X) .
$$

Let $N_{1}, N_{2}$ and $N_{3}$ be modules such that:

- $N_{1}$ is a quotient of $\mathrm{E}^{d-2}(X)$ (which is torsion free so that $\mathrm{E}^{d-2}\left(N_{1}\right)=0$ );

- $N_{2}$ is a submodule of $\mathrm{E}^{d-1}(X)$ (which is finite so that $\mathrm{E}^{d-1}\left(N_{2}\right)=0$ );

- $N_{3}$ is a module such that the sequences

$$
N_{1} \hookrightarrow \bigoplus_{S} \operatorname{Ind}_{G_{v}}^{G} \mathrm{E}_{v}^{d-2}\left(X_{v}\right) \rightarrow N_{3} \quad \text { and } \quad N_{3} \hookrightarrow \mathrm{E}^{d-1}(\mathcal{S}) \rightarrow N_{2}
$$

are exact.

Applying the functor Ext we find

$$
\mathrm{E}^{d-2}\left(N_{1}\right) \rightarrow \mathrm{E}^{d-1}\left(N_{3}\right) \rightarrow \bigoplus_{S} \operatorname{Ind}_{G_{v}}^{G} \mathrm{E}_{v}^{d-1} \mathrm{E}_{v}^{d-2}\left(X_{v}\right)
$$

(which yields $\mathrm{E}^{d-1}\left(N_{3}\right)=0$ ), and

$$
\mathrm{E}^{d-1}\left(N_{2}\right) \rightarrow \mathrm{E}^{d-1} \mathrm{E}^{d-1}(\mathcal{S}) \rightarrow \mathrm{E}^{d-1}\left(N_{3}\right)
$$

which proves $\mathrm{E}^{d-1} \mathrm{E}^{d-1}(\mathcal{S})=0$.

Remark 4.2. As pointed out in various steps of the previous proof, most of the statements still hold for $d=1,2$. The only missing part is $\mathrm{E}^{2}(\operatorname{Ker}(f))=0$ for $i=2$, in that case only our calculations to get $\mathrm{E}^{2} \mathrm{E}^{2}(\mathcal{S})=0$ fail. In particular the same proof shows that $\mathrm{E}^{2} \mathrm{E}^{2}(\mathcal{S})=0$ when $\operatorname{Ker}(f)$ is free and $d=1$ or when $\operatorname{Ker}(f)$ is finite and $d=2$ or, obviously, for any $d$ if $f$ is injective. 
We can extend the previous result to the $d \geqslant 2$ case with some extra assumptions.

Proposition 4.3. Let $G=\operatorname{Gal}(K / F)$ be an $\ell$-adic Lie group without elements of order $\ell$ and of dimension $d \geqslant 2$. If $H^{2}\left(F_{S} / K, A\left[\ell^{\infty}\right]\right)=0$ and $\operatorname{cd}_{\ell}\left(G_{v}\right)=2$ for any $v \in S$, then $\operatorname{Sel}_{A}(K)_{\ell}^{\vee}$ has no nontrivial pseudo-null submodule.

Proof. Since $\operatorname{cd}_{\ell}\left(F_{v}\right)=2$ (by [NSW, Theorem 7.1.8]), our hypothesis implies that $\operatorname{Gal}\left(\overline{F_{v}} / K_{w}\right)$ has no elements of order $l$ (see also [NSW, Theorem 7.5.3]). Hence $H^{1}\left(K_{w}, A\left[\ell^{\infty}\right]\right)^{\vee}=0$ and $\operatorname{Sel}_{A}(K)_{\ell}^{\vee} \simeq X$ embeds in $Y$. Now $H^{2}\left(F_{S} / K, A\left[\ell^{\infty}\right]\right)=0$ yields $Y$ has projective dimension $\leqslant 1$, so $Y$ has no nontrivial pseudo-null submodule (by [OV, Proposition 2.5]).

4.1. The hypotheses on $H^{2}\left(F_{S} / K, A\left[\ell^{\infty}\right]\right)$ and $\psi$. Let $F_{m}$ be extensions of $F$ such that $\operatorname{Gal}(K / F) \simeq \lim _{\overleftarrow{m}} \operatorname{Gal}\left(F_{m} / F\right)$. To provide some cases in which the main hypotheses hold we consider the Poitou-Tate sequence for the module $A\left[\ell^{n}\right]$, from which one can extract the sequence

$$
\begin{aligned}
& 0 \longrightarrow \operatorname{Ker}\left(\psi_{m, n}\right) \longrightarrow H^{1}\left(F_{S} / F_{m}, A\left[\ell^{n}\right]\right) \stackrel{\psi_{m, n}}{\longrightarrow} \prod_{v_{v \in S} \mid v} H^{1}\left(F_{v_{m}}, A\left[\ell^{n}\right]\right) \\
& \left.\prod_{\substack{v_{m} \mid v \\
v \in S}} H^{2}\left(F_{v_{m}}, A\left[\ell^{n}\right]\right) \longleftarrow H^{2}\left(F_{S} / F_{m}, A\left[\ell^{n}\right]\right) \longleftarrow \operatorname{Ker}\left(\psi_{m, n}^{t}\right)\right)^{\vee} \\
& \downarrow \\
& H^{0}\left(F_{S} / F_{m}, A^{t}\left[\ell^{n}\right]\right)^{\vee} \longrightarrow 0
\end{aligned}
$$

(where $\psi_{m, n}^{t}$ is the analogue of $\psi_{m, n}$ for the dual abelian variety $A^{t}$, i.e., their kernels represent the Selmer groups over $F_{m}$ for the modules $A^{t}\left[\ell^{n}\right]$ and $A\left[\ell^{n}\right]$ respectively). Taking direct limits on $n$ and recalling that $H^{2}\left(F_{v_{m}}, A\left[\ell^{\infty}\right]\right)=0$, the sequence (17) becomes

$$
\begin{aligned}
& 0 \longrightarrow \operatorname{Sel}_{A}\left(F_{m}\right)_{\ell} \longrightarrow H^{1}\left(F_{S} / F_{m}, A\left[\ell^{\infty}\right]\right) \stackrel{\psi_{m}}{\longrightarrow} \prod_{\substack{v_{m} \mid v \\
v \in S}} H^{1}\left(F_{v_{m}}, A\left[\ell^{\infty}\right]\right) \\
& 0 \longleftarrow H^{2}\left(F_{S} / F_{m}, A\left[\ell^{\infty}\right]\right) \longleftarrow\left(\lim _{n} \operatorname{Ker}\left(\psi_{m, n}^{t}\right)\right)^{\vee}
\end{aligned}
$$

(for more details one can consult [CS, Chapter 1]). One way to prove that $H^{2}\left(F_{S} / K, A\left[\ell^{\infty}\right]\right)=$ 0 and $\psi$ is surjective is to show that $\left.\underset{\leftarrow}{\lim } \operatorname{Ker}\left(\psi_{m, n}^{t}\right)\right)^{\vee}=0$ for any $m$. We mention here two cases in which the hypothesis on the vanishing of $H^{2}\left(F_{S} / K, A\left[\ell^{\infty}\right]\right)$ is verified. The following is basically [CS, Proposition 1.9].

Proposition 4.4. Let $F_{m}$ be as above and assume that $\left|S e l_{A^{t}}\left(F_{m}\right)_{\ell}\right|<\infty$ for any $m$, then

$$
H^{2}\left(F_{S} / K, A\left[\ell^{\infty}\right]\right)=0 \text {. }
$$

Proof. From [M, Chapter I Remark 3.6 ] we have the isomorphism

$$
A^{t}\left(F_{v_{m}}\right)^{*} \simeq H^{1}\left(F_{v_{m}}, A\left[\ell^{\infty}\right]\right)^{\vee},
$$

where $A^{t}\left(F_{v_{m}}\right)^{*} \simeq \lim _{\leftarrow} A^{t}\left(F_{v_{m}}\right) / \ell^{n} A^{t}\left(F_{v_{m}}\right)$.

Taking inverse limits on $n$ in the exact sequence

$$
A^{t}\left(F_{m}\right) / \ell^{n} A^{t}\left(F_{m}\right) \hookrightarrow \operatorname{Ker}\left(\psi_{m, n}^{t}\right) \rightarrow \amalg\left(A^{t} / F_{m}\right)\left[\ell^{n}\right],
$$

and noting that $\left|\amalg\left(A^{t} / F_{m}\right)\left[\ell^{\infty}\right]\right|<\infty$ yields $T_{\ell}\left(\amalg\left(A^{t} / F_{m}\right)\right)=0$, we find

$$
A^{t}\left(F_{m}\right)^{*} \simeq \lim _{n} \operatorname{Ker}\left(\psi_{m, n}^{t}\right) \text {. }
$$


Therefore (18) becomes

$$
\begin{aligned}
& 0 \longrightarrow \operatorname{Sel}_{A}\left(F_{m}\right)_{\ell} \longrightarrow H^{1}\left(F_{S} / F_{m}, A\left[\ell^{\infty}\right]\right) \stackrel{\psi}{\longrightarrow} \prod_{\substack{v_{m} \mid v \\
v \in S}}\left(A^{t}\left(F_{v_{m}}\right)^{*}\right)^{\vee} \\
& \downarrow \widetilde{\phi} \\
& 0 \longleftarrow H^{2}\left(F_{S} / F_{m}, A\left[\ell^{\infty}\right]\right) \longleftarrow\left(A^{t}\left(F_{m}\right)^{*}\right)^{\vee}
\end{aligned}
$$

By hypothesis $A^{t}\left(F_{m}\right)^{*}$ is finite, therefore $H^{2}\left(F_{S} / F_{m}, A\left[\ell^{\infty}\right]\right)$ is finite as well. From the cohomology of the sequence

$$
A[\ell] \hookrightarrow A\left[\ell^{\infty}\right] \stackrel{\ell}{\longrightarrow} A\left[\ell^{\infty}\right]
$$

(and the fact that $H^{3}\left(F_{S} / F_{m}, A[\ell]\right)=0$, because $c d_{\ell}\left(\operatorname{Gal}\left(F_{S} / F_{m}\right)\right)=2$ ), one finds

$$
H^{2}\left(F_{S} / F_{m}, A\left[\ell^{\infty}\right]\right) \stackrel{\ell}{\longrightarrow} H^{2}\left(F_{S} / F_{m}, A\left[\ell^{\infty}\right]\right),
$$

i.e., $H^{2}\left(F_{S} / F_{m}, A\left[\ell^{\infty}\right]\right)$ is divisible. Being divisible and finite $H^{2}\left(F_{S} / F_{m}, A\left[\ell^{\infty}\right]\right)$ must be 0 for any $m$ and the claim follows.

We can also prove the vanishing of $H^{2}\left(F_{S} / K, A\left[\ell^{\infty}\right]\right)$ for the extension $K=F\left(A\left[\ell^{\infty}\right]\right)$.

Proposition 4.5. If $K=F\left(A\left[\ell^{\infty}\right]\right)$, then $H^{2}\left(F_{S} / K, A\left[\ell^{\infty}\right]\right)=0$.

Proof. $\operatorname{Gal}\left(F_{S} / K\right)$ has trivial action on $A\left[\ell^{\infty}\right]$ and (by the Weil pairing) on $\boldsymbol{\mu}_{\ell^{\infty}}$, so

$$
H^{2}\left(F_{S} / K, A\left[\ell^{\infty}\right]\right) \simeq H^{2}\left(F_{S} / K,\left(\mathbb{Q}_{\ell} / \mathbb{Z}_{\ell}\right)^{2 g}\right) \simeq H^{2}\left(F_{S} / K,\left(\boldsymbol{\mu}_{\ell \infty}\right)^{2 g}\right) .
$$

Let $F_{n}=F\left(A\left[\ell^{n}\right]\right)$, using the notations of Lemma 3.6, Poitou-Tate duality ([NSW, Theorem 8.6.7]) and the isomorphism $\amalg^{1}\left(F_{S} / F_{n}, \mathbb{Z} / \ell^{m} \mathbb{Z}\right) \simeq \operatorname{Hom}\left(C \ell_{S}\left(F_{n}\right), \mathbb{Z} / \ell^{m} \mathbb{Z}\right)$ ([NSW, Lemma 8.6.3]), one has

$$
\begin{aligned}
H^{2}\left(F_{S} / K, \boldsymbol{\mu}_{\ell \infty}\right) & \simeq \amalg^{2}\left(F_{S} / K, \boldsymbol{\mu}_{\ell^{\infty}}\right) \simeq \lim _{\overrightarrow{n, m}} \amalg^{2}\left(F_{S} / F_{n}, \boldsymbol{\mu}_{\ell^{m}}\right) \\
& \simeq \lim _{\overrightarrow{n, m}} \amalg^{1}\left(F_{S} / F_{n}, \boldsymbol{\mu}_{\ell^{m}}^{\prime}\right)^{\vee} \simeq \lim _{\overrightarrow{n, m}} \amalg^{1}\left(F_{S} / F_{n}, \mathbb{Z} / \ell^{m} \mathbb{Z}\right)^{\vee} \\
& \simeq \lim _{\overrightarrow{n, m}} \operatorname{Hom}\left(C \ell_{S}\left(F_{n}\right), \mathbb{Z} / \ell^{m} \mathbb{Z}\right)^{\vee} \simeq \lim _{\overrightarrow{n, m}} C \ell_{S}\left(F_{n}\right) / \ell^{m} \\
& \simeq \lim _{\vec{n}} C \ell_{S}\left(F_{n}\right) \otimes_{\mathbb{Z}} \mathbb{Q}_{\ell} / \mathbb{Z}_{\ell}=0
\end{aligned}
$$

since $C \ell_{S}\left(F_{n}\right)$ is finite.

Remark 4.6. The above proposition works in the same way for a general $\ell$-adic Lie extensions, unramified outside $S$, which contains the trivializing extension.

Example 4.7. Let $A$ be an abelian variety without complex multiplication: by Proposition 4.5, the extension $K=F\left(A\left[\ell^{\infty}\right]\right)$ realizes the hypothesis of Proposition 4.3 when every bad reduction prime is of split multiplicative reduction (in order to have $\operatorname{cd}_{\ell}\left(G_{v}\right)=2$ ) and $\ell>$ $2 g+1$ (by $[\mathrm{ST}]$ and the embedding $\operatorname{Gal}(K / F) \hookrightarrow \mathrm{GL}_{2 g}\left(\mathbb{Z}_{\ell}\right)$ ). Therefore $\operatorname{Sel}_{A}(K)_{\ell}^{\vee}$ has no nontrivial pseudo-null submodule. When $A=\mathcal{E}$ is an elliptic curve (using Igusa's theorem, see, e.g., $[\mathrm{BLV}])$ one can prove that $\operatorname{dim} \operatorname{Gal}(K / F)=4$ and also the surjectivity of the map $\psi$ (which, in this case, is not needed to prove the absence of pseudo-null submodules): more details can be found in $[\mathrm{S}]$.

The same problem over number fields cannot (in general) be addressed in the same way and one needs the surjectivity of the map $\psi$. The topic is treated (for example) in [C, Section 4.2] and [HV, Section 7.1]. 


\section{REFERENCES}

[BBL] A. Bandini, F. Bars and I. Longhi Aspects of Iwasawa theory over function fields. To appear in the EMS Congress Reports, arXiv:1005.2289v2 [math.NT] (2010).

[BL] A. Bandini and I. Longhi Control theorems for elliptic curves over function fields. Int. J. Number Theory 5 no. 2 (2009), 229-256.

[BL2] A. Bandini and I. Longhi Selmer groups for elliptic curves in $\mathbb{Z}_{l}^{d}$-extensions of function fields of characteristic p. Ann. Inst. Fourier 59 no. 6 (2009), 2301-2327.

$[\mathrm{BLV}]$ A. Bandini, I. Longhi and S. Vigni. Torsion points on elliptic curves over function fields and a theorem of Igusa. Expo. Math. 27 no. 3 (2009), 175-209.

[BV] A. Bandini and M. Valentino. Control Theorems for $\ell$-adic Lie extensions of global function fields. To appear in Ann. Sc. Norm. Sup. Pisa Cl. Sci. DOI Number: 10.2422/2036-2145.201304_001 , arXiv:1206.2767v2 [math.NT] (2012).

[B] N. Bourbaki. Commutative Algebra. Paris: Hermann (1972).

[C] J. Coates. Fragments of the $G L_{2}$ Iwasawa theory of elliptic curves without complex multiplication. in Arithmetic Theory of Elliptic Curves, (Cetraro, 1997), Ed. C. Viola, Lecture Notes in Mathematics 1716, Springer (1999), 1-50.

[CS] J. Coates and R. Sujatha. Galois cohomology of elliptic curves. 2nd Edition. Narosa (2010).

[DdSMS] J.D. Dixon, M.P.F. du Sautoy, A. Mann and D. Segal. Analytic pro-p groups. 2nd Edition. Cambridge Studies in Advanced Mathematics 61, Cambridge Univ. Press (1999).

[HV] O. Hachimori and O. Venjakob Completely faithful Selmer groups over Kummer extensions. Doc. Math. Extra Vol. Kazuya Kato's fiftieth birthday (2003), 443-478.

[HO] O. Hachimori and T. Ochiai Notes on non-commutative Iwasawa theory. Asian J. Math. 14 no. 1 (2010), 11-17.

[J] U. Jannsen. Iwasawa modules up to isomorphism. Advanced Studies in Pure Mathematics 17 (1989), Algebraic Number Theory - in honor of K. Iwasawa, 171-207.

[J1] U. Jannsen. A spectral sequence for Iwasawa adjoints, arXiv:1307.6547v1 [math.NT] (2013).

[L] M. Lazard. Groupes analytiques p-adiques. Publ. Math. I.H.E.S. 26 (1965), 389-603.

[M] J.S. Milne. Arithmetic Duality Theorems. BookSurge, LLC, Second edition, 2006.

[NSW] J. Neuchirch, A. Schmidt and K. Wingberg. Cohomology of number fields - Second edition. GTM 323, Springer-Verlag, (2008).

[O] Y. Ochi. A note on Selmer groups of abelian varieties over the trivializing extensions. Proc. Amer. Math. Soc. 134 no. 1 (2006), 31-37 .

[OV] Y. Ochi and O. Venjakob. On the structure of Selmer groups over $p$-adic Lie extensions. J. Algebraic Geom. 11 no. 3 (2002), 547-580.

[OT] T. Ochiai and F. Trihan, On the Selmer groups of abelian varieties over function fields of characteristic $p>0$, Math. Proc. Cambridge Philos. Soc. 146 no. 1 (2009), 23-43.

[S] G. Sechi. GL Iwasawa Theory of Elliptic Curves over Global Function Fields. PhD thesis, University of Cambridge, (2006).

[Se] J.P. Serre. Sur la dimension cohomologique des groupes profinis. Topology 3 (1965), 413-420.

[ST] J.P. Serre and J. Tate. Good reduction of abelian varieties. Ann. of Math. 88 (1968), 492-517.

[T] K.-S. Tan A generalized Mazur's theorem and its applications. Trans. Amer. Math. Soc. 362 no. 8 (2010), 4433-4450.

[V] O. Venjakob. On the structure theory of the Iwasawa algebra of a p-adic Lie group. J. Eur. Math. Soc. (JEMS) 4 no. 3 (2002), 271-311.

[W] M. Witte, On a noncommutative Iwasawa main conjecture for varieties over finite fields. to appear in J. Eur. Math. Soc. (JEMS), arXiv:1004.2481v2 [math.NT] (2012).

Andrea Bandini

Università degli Studi di Parma - Dipartimento di Matematica e Informatica

Parco Area delle Scienze, 53/A - 43124 Parma - Italy

e-mail: andrea.bandini@unipr.it

Maria Valentino

Università della Calabria - Dipartimento di Matematica e Informatica

via P. Bucci - Cubo 31B - 87036 Arcavacata di Rende (CS) - Italy

e-mail: maria.valentino84@gmail.com 